\section{Medicina Baseada em Evidências}

\section{TratameNTO DA OTITE MÉDIA AGUDA NA INFÂNCIA}

Autoria: Departamento de Otorrinolaringologia da Sociedade Brasileira de Pediatria e Academia de Otorrinolaringologia Pediátrica da Associação Brasileira de Otorrinolaringologia e Cirurgia Cérvico-facial

Participantes: Eulália Sakano, Luc L. M. Weckx, Wanderley M. Bernardo, Moacyr Saffer

Método de coleta de evidências: Foram consultadas as bases de dados Medline, através do Pubmed, a base de dados Cochrane de Revisões Sistemáticas e o Registro de Ensaios Controlados da Colaboração Cochrane, por meio da BVS. A estratégia de busca utilizada baseou-se em perguntas estruturadas na forma de PICO (Paciente, Intervenção, Controle, Outcome). A sintaxe de busca resultante foi: ("Otitis Media" AND Acute Disease) AND (Amoxicillin OR AntiBacterial Agents OR Clavulanic Acid OR Azithromycin OR Clarithromycin OR Cefaclor OR Cephalosporins), Field: All Fields, Limits: All Child: 0- 18 years, recuperando 799 artigos. Foram então acrescidos os filtros: Randomized Controlled Trial, Meta-Analysis, resultando em I 85 publicações. A seguir foram selecionados pelo abstract os 107 trabalhos relacionados diretamente às questões clínicas. Esses trabalhos tiveram a sua força de evidência científica classificada segundo as normas da Oxford Centre for Evidence Based Medicine(www.projetodiretrizes.org.br), como também os ensaios clínicos controlados e randomizados foram submetidos à avaliação crítica'(A). Por fim, selecionaram-se as 17 referências que, pela maior força de evidência científica, consistência e relevância clínica, deram a sustentação às recomendações da presente diretriz.

Graus de recomendação e força de evidência científica:

A: Estudos experimentais ou observacionais de melhor consistência.

B: Estudos experimentais ou observacionais de menor consistência.

C: Relatos de casos (estudos não controlados).

D: Opinião desprovida de avaliação crítica, baseada em consensos, estudos fisiológicos ou modelos animais.

\section{Deve-se utilizar antibióticos no tratamento da otite média (OM) em crianças?}

Crianças não tratadas não têm resolução da dor, nas primeiras 24 horas, em $41 \%$ dos casos (NNTH: 2). E crianças não tratadas não têm resolução da dor e da febre, entre o segundo e sétimo dia, em 13\% dos casos (NNTH: 8) ${ }^{8}(\mathbf{A})$.

$\mathrm{O}$ uso de antibióticos reduz a dor de maneira menos importante nas primeiras 24 horas (NNT: I 5) e de maneira mais acentuada no período de dois a sete dias (NNT:5). A recorrência, timpanometria e complicações não têm diferença significativa. Os antibióticos reduzem o curso da otite média aguda (OMA) e da progressão para otite bilateral ${ }^{2}(\mathbf{A})$.

A presença de temperatura $t$ 37,5, tosse e vômitos no primeiro dia de OMA está relacionada com maior incidência de desconforto e alteração do sono no terceiro dia. $O$ uso imediato de antibióticos nessas crianças reduz essa ocorrência ${ }^{3}(\mathbf{A})$.

O uso imediato, nas primeiras 24 horas, de antibiótico, em crianças com OMA, reduz o tempo de doença, produz menos distúrbios noturnos e menor ingestão de analgésicos (paracetamol) ${ }^{4}(\mathbf{A})$.

Crianças não tratadas têm piora clínica em quatro a sete dias, em $22 \%$ dos casos (NNTH:5) ${ }^{7}(\mathbf{A})$.

\section{A amoxicilina é ainda a primeira opção no tratamento da OMA?}

A resposta clínica da OMA ao tratamento com amoxicilina $(60 \mathrm{mg} / \mathrm{kg} / \mathrm{d})$ é superior ao tratamento com placebo (NNT: I I). Há menor incidência de febre (NNT: 6) e dor nos primeiros dois dias em pacientes tratados com amoxicilina. Em relação aos eventos adversos, não há diferença significativa entre amoxicilina e placebo ${ }^{5}(\mathbf{A})$.

Não há diferença entre a resposta clínica ao tratamento com amoxicilina $(90 \mathrm{mg} / \mathrm{kg} / \mathrm{d})$ e azitromicina $(30 \mathrm{mg} / \mathrm{kg})(\mathrm{NNT}: \mathrm{II})$. Há melhor resposta clínica ao tratamento com amoxicilina $(90 \mathrm{mg} / \mathrm{kg} / \mathrm{d})$ nos casos de associação de $S$. pneumoniae e $H$. influenzae (NNT: I,3) ${ }^{6}(\mathbf{A})$.

É necessário tratar oito crianças com ampicilina ou amoxicilina para se evitar uma evolução clínica inadequada (falha clínica). A amoxicilina não apresenta diferença no resultado terapêutico quando comparada à penicilina, cefaclor e cefixime ${ }^{7}(\mathbf{A})$.

O tratamento da OMA com amoxicilina reduz o risco relativo de persistência dos sintomas, no quarto dia, em $18 \%$, quando comparado ao placebo (NNT: 6). Não há diferença no índice de falha terapêutica no $11^{\circ}$ dia de OMA, no tratamento com amoxicilina em relação ao placebo ${ }^{9}(\mathbf{A})$.

A amoxicilina está indicada no primeiro episódio de $\mathrm{OMA}^{14}(\mathbf{B})$.

\section{Há indicação para a amoxicilina em dose dobrada no tratamento da OMA?}

$O$ uso de dose alta de amoxicilina não confere melhor resposta clínica, menor recorrência ou eventos adversos do que a dose padrão. Por semelhante modo, não há diferença entre duas ou três tomadas de amoxicilina ${ }^{7}(\mathbf{A})$.

Não há diferença entre a resposta clínica ao tratamento com amoxicilina (90 mg/kg/d) e amoxicilina (40-45 mg/ $/ \mathrm{kg} / \mathrm{d}$ ), em crianças maiores que três meses (IC: $-1,5 \%$ a 3,4\%) "'(A).

\section{Quando indicar a amoxiclavulanato no tratamento da} OMA?

Em crianças de três meses a três anos com IVAS e história prévia de $O \mathrm{MA}^{12}(\mathbf{A})$ :

- É necessário tratar I 5 crianças com amoxiclavulanato $(75 \mathrm{mg} /$ $\mathrm{kg} / \mathrm{d}$ ) para se evitar uma OMA, quando comparado ao placebo; 
- É necessário tratar 10 crianças com amoxiclavulanato $(75 \mathrm{mg} /$ $\mathrm{kg} / \mathrm{d}$ ) para se evitar um uso de paracetamol, quando comparado ao placebo;

- Não há diferença significativa de efeitos adversos.

Não há diferença entre a resposta clínica ao tratamento com amoxiclavulanato $(90 \mathrm{mg} / 6,4 \mathrm{mg} / \mathrm{kg} / \mathrm{d})$ e azitromicina $(20 \mathrm{mg} /$ $\mathrm{kg} / \mathrm{d})$ nas primeiras duas semanas após tratamento ${ }^{13}(\mathbf{A})$.

Não há diferença entre a resposta clínica ao tratamento com amoxiclavulanato $(45 \mathrm{mg} / \mathrm{kg} / \mathrm{d})$ e azitromicina $(10 \mathrm{mg} / \mathrm{kg} / \mathrm{d})$. Mas em relação às diferenças de sinais presentes na membrana timpânica, nessas duas modalidades terapêuticas, verificamos ${ }^{10}(\mathbf{A})$ :

- No $30^{\circ}$ dia, o abaulamento (NNT: 17) e o prejuízo de motilidade (NNT: 13) estão mais presentes nos pacientes tratados com amoxicilina;

- No $10^{\circ}$ dia, o abaulamento e o prejuízo de motilidade estão mais presentes nos pacientes tratados com azitromicina (NNT: 12).

\section{A azitromicina pode ser utilizada no tratamento da OMA?}

Nos primeiros três dias de tratamento, a azitromicina tem maior falha terapêutica do que a amoxicilina (OR: I,6). A azitromicina está associada a menor falha terapêutica em relação à amoxicilina (OR: 0,88), após 30 dias do tratamento ${ }^{14}(\mathbf{B})$.

Entretanto, metaanálise de ensaios clínicos controlados randomizados, que avaliaram a eficácia e a segurança da azitromicina em relação a outros antibióticos, nas infecções do trato respiratório superior, não encontrou diferença de falha terapêutica global na OMA, quando comparada a azitromicina com amoxiclavulanato (dez estudos incluídos), cefaclor (quatro estudos), claritromicina (um estudo) e amoxicilina (um estudo) ${ }^{15}(\mathbf{A})$.

A azitromicina apresenta menos eventos adversos do que a amoxicilina (NNT: 10) ${ }^{6}(\mathbf{A})$. É necessário tratar oito crianças com azitromicina para se evitar um evento adverso gastrointestinal, quando comparado à amoxicilina ${ }^{8}(\mathbf{A})$.

No $30^{\circ}$ dia e nas crianças menores de dois anos, a resposta foi superior na azitromicina $(20 \mathrm{mg} / \mathrm{kg} / \mathrm{d})$ do que no amoxiclavulanato $(90 \mathrm{mg} / 6,4 \mathrm{mg} / \mathrm{kg} / \mathrm{d})(\mathrm{NNT}: 6)^{13}(\mathbf{A})$.

\section{Há indicação para o uso de corticóide oral, descon- gestionante, AINH e analgésicos no tratamento da OMA?}

Devido ao pequeno benefício e ao risco aumentado de efeitos adversos, o uso de descongestionantes e anti-histamínicos não é recomendado para crianças com otite média aguda $^{16}(\mathbf{A})$.

O tratamento de cinco dias com anti-histamínicos ou corticóide em associação com antibióticos não melhora os resultados terapêuticos na OMA. Os anti-histamínicos são contra-indicados para crianças com otite média aguda, pois prolongam o tempo de líquido no $\mathrm{OM}^{17}(\mathbf{A})$.
O ibuprofeno mostrou ser superior ao placebo na resolução da $\operatorname{dor}^{18}(\mathbf{A})$.

Referências

I.Jadad AR, Moore RA, Carroll D, Jenkinson C, Reynolds DJ, Gavaghan DJ, McQuay HJ. Assessing the quality of reports of randomized clinical trials: is blinding necessary? Control Clin Trials 1996; I7: I - I2.

2.Glasziou PP, Del Mar CB, Sanders SL, Hayem M. Antibiotics for acute otitis media in children. Cochrane Database Syst Rev 2004;( I ):CD0002 19.

3.Little P, Gould C, Moore M, Warner G, Dunleavey J, Williamson I. Predictors of poor outcome and benefits from antibiotics in children with acute otitis media: pragmatic randomised trial. BMJ 2002;325:22.

4.Little P, Gould C, Williamson I, Moore M, Warner G, Dunleavey J. Pragmatic randomised controlled trial of two prescribing strategies for childhood acute otitis media. BMJ 2001;322:336-42.

5.Le Saux N, Gaboury I, Baird M, Klassen TP, MacCormickJ, Blanchard C, et al. A randomized, double-blind, placebo-controlled noninferiority trial of amoxicillin for clinically diagnosed acute otitis media in children 6 months to 5 years of age. CMAJ 2005; I 72:335-4I.

6.Arguedas A, Emparanza P, Schwartz RH, Soley C, Guevara S, de Caprariis PJ, et al. A randomized, multicenter, double blind, double dummy trial of single dose azithromycin versus high dose amoxicillin for treatment of uncomplicated acute otitis media. Pediatr Infect Dis J 2005; 24:| 53-6|.

7.Takata GS, Chan LS, Shekelle P, Morton SC, Mason W, Marcy SM. Evidence assessment of management of acute otitis media: I. The role of antibiotics in treatment of uncomplicated acute otitis media. Pediatrics $2001 ; 108: 239-47$

8.Marcy M, Takata G, Chan LS, Shekelle P, Mason W, Wachsman L, et al. Management of acute otitis media. Evid Rep Technol Assess (Summ). 2000; I 5: I -4. 9.Damoiseaux RA, van Balen FA, Hoes AW, Verheij TJ, de Melker RA. Primary care based randomised, double blind trial of amoxicillin versus placebo for acute otitis media in children aged under 2 years. BMJ 2000; 320:350-4.

10.Dunne MW, Latiolais T, Lewis B, Pistorius B, Bottenfield G, Moore WH, et al. Randomized, double-blind study of the clinical efficacy of 3 days of azithromycin compared with co-amoxiclav for the treatment of acute otitis media. J Antimicrob Chemother 2003;52:469-72.

I I.Garrison GD, Sorum PC, Hioe W, Miller MM. High-dose versus standarddose amoxicillin for acute otitis media. Ann Pharmacother 2004;38: I 5-9.

12.Autret-Leca E, Giraudeau B, Ployet MJ, Jonville-Bera AP. Amoxicillin/ clavulanic acid is ineffective at preventing otitis media in children with presumed viral upper respiratory infection: a randomized, double-blind equivalence, placebo-controlled trial. Br J Clin Pharmacol. 2002;54:652-6.

13.Arrieta A, Arguedas A, Fernandez P, Block SL, Emperanza P, Vargas SL, et al. High-dose azithromycin versus high-dose amoxicillin-clavulanate for treatment of children with recurrent or persistent acute otitis media. Antimicrob Agents Chemother 2003;47:3179-86.

14.Quach C, Collet JP, LeLorier J. Effectiveness of amoxicillin, azithromycin, cefprozil and clarithromycin in the treatment of acute otitis media in children: a population-based study. Pharmacoepidemiol Drug Saf 2005; 14: $163-70$.

I 5.loannidis JP, Contopoulos-loannidis DG, Chew P, Lau J. Meta-analysis of randomized controlled trials on the comparative efficacy and safety of azithromycin against other antibiotics for upper respiratory tract infections. J Antimicrob Chemother 200 I;48:677-89.

1 6. Flynn CA, Griffin GH, Schultz JK. Decongestants and antihistamines for acute otitis media in children. Cochrane Database Syst Rev 2004; (3):CD00 1727.

17.Chonmaitree T, Saeed K, Uchida T, Heikkinen T, Baldwin CD, Freeman DH $\mathrm{J}$, et al. A randomized, placebo-controlled trial of the effect of antihistamine or corticosteroid treatment in acute otitis media. J Pediatr 2003; | 43:377-85.

18. Bertin L, Pons G, d'Athis P, Duhamel JF, Maudelonde C, Lasfargues G, et al. A randomized, double-blind, multicentre controlled trial of ibuprofen versus acetaminophen and placebo for symptoms of acute otitis media in children. Fundam Clin Pharmacol 1996; 10:387-92. 